\title{
Lettera al Direttore
}

Spett. SEEd editore,

in relazione alla review dal titolo "Entecavir nella terapia dell'epatite B cronica: profilo farmacoeconomico" pubblicata sul numero 2011; 12(2) della vostra rivista, desideriamo manifestare alcune perplessità, in particolare nella sezione farmaco economica.

L'Autore a pagina 69 cita un modello di Markov quale dimostrazione del significativo progresso terapeutico ottenuto con l'impiego di Entecavir nell'epatite cronica B (ref. 57). Tuttavia, tale riferimento bibliografico non è costituito da un lavoro pubblicato, ma da "data on file" dell'azienda BMS e pertanto, a nostro avviso, non possiede i requisiti per essere considerato un testo scientificamente condiviso.

Lo stesso modello citato non viene poi né presentato né discusso nel testo; ciò preclude la possibilità di interpretarlo e di comprenderne i risultati finali. Non disponendo pertanto dei dati e delle premesse metodologiche cui l'Autore fa riferimento, ma dei soli dati di letteratura, non ci è stato possibile effettuare alcuna verifica sul modello e soprattutto delle implicazioni dello stesso. Queste ultime costituiscono peraltro una significativa parte dell'articolo, che resta visibilmente incongruente con quanto osservato in precedenza. A pagina 70 l'Autore propone, senza adeguata spiegazione metodologica che lo giustifichi, un confronto tra entecavir e tenofovir; come noto le possibili strategie di trattamento dell'epatite cronica B non si esauriscono a questi due farmaci, ma si aprono a scenari di trattamento ben più complessi. In particolare ciò che non è chiaro è su quali basi venga effettuato il confronto, ovvero se sia stato realizzato utilizzando il precedente modello o ne sia stato predisposto uno nuovo.

Verso la fine dell'articolo viene presentato il problema dei costi di monitoraggio renale e dei costi di gestione di un'eventuale reazio- ne avversa severa durante trattamento con tenofovir. Al di là della loro valorizzazione, nel testo non emerge in alcun modo che i costi di gestione di una reazione avversa a una terapia sono il prodotto del costo di gestione dell'evento moltiplicato per la frequenza relativa con cui lo stesso accade. Poiché la frequenza con cui avviene un evento avverso severo renale, quale quello citato in Tabella VIII, è negli studi registrativi e in quelli di pratica clinica sostanzialmente uguale a zero, riteniamo fuorviante che vengano riportati i DRG della gestione dei pazienti con insufficienza renale.

Concordiamo sulle conclusioni di pagina 73 , dove l'Autore afferma che: «ad oggi, non è disponibile una valutazione precisa e robusta dell'impatto sul budget sanitario di entecavir vs. tenofovir, anche in virtù della mancanza di studi clinici controllati di confronto diretto tra i due farmaci». Tuttavia, a fronte di questa constatazione, non si può non citare la recente letteratura di confronto tra i due farmaci. E ciò nonostante l'Autore inoltre si spinge ad affermare che sulla base del modello riportato nell'articolo (non presentato né discusso) entecavir rappresenta una scelta favorevole in termini di costo per trattamento ed eventi avversi evitati. Riteniamo che tale assunto richieda di essere inserito in un apparato scientifico di dati e letteratura molto più significativo di quanto non sia quello riportato in questo articolo.

Cordialmente,

Giorgio L. Colombo, Università degli Studi di Pavia, Facoltà di Farmacia e S.A.V.E. Studi Analisi Valutazioni Economiche, Via Previati, 74 - 20149 Milano, Italia

Mauro Viganò, Unità di Epatologia, Ospedale San Giuseppe, Milano e Università degli Studi di Milano Via San Vittore 12 - 20123 Milano, Italia 


\section{BIBLIOGRAFIA}

- Colombo GL, Gaeta GB, Viganò M, et al. A cost-effectiveness analysis of different therapies in patients with chronic hepatitis B in Italy. Clinicoecon Outcomes Res 2011; 3: 37-46

- European Association For The Study Of The Liver. EASL Clinical Practice Guidelines: management of chronic hepatitis B. J Hepatol 2009; 50: 227-42

- Idris BI, Brosa M, Richardus JH, et al. Estimating the future health burden of chronic hepatitis B and the impact of therapy in Spain. Eur J Gastroenterol Hepatol 2008; 20: 320-6

- Heathcote JE, Gane EJ, De Man A, et al. Three years of tenofovir disoproxil (TDF) treatment in HbeAg-positive patients $(\mathrm{HBeAg}+)$ with chronic hepatitis B (Study 103), preliminary analysis. [Abstract]. Hepatology 2009; 50(Suppl 4): 533A

- Chang TT, Gish RG, de Man R, et al. A comparison of entecavir and lamivudine for HBeAg-positive chronic hepatitis B. N Engl J Med 2006; 354: 1001-10

- Lai CL, Yuen MF. Chronic hepatitis B--new goals, new treatment. N Engl J Med 2008; 359: 2488-91

- Tenney DJ, Rose RE, Baldick CJ, et al. Long-term monitoring shows hepatitis B virus resistance to entecavir in nucleoside-naïve patients is rare through 5 years of therapy. Hepatology 2009; 49: 1503-14

- Liaw YF, Gane E, Leung N, et al. GLOBE Study Group. 2-Year GLOBE trial results: telbivudine Is superior to lamivudine in patients with chronic hepatitis B. Gastroenterology 2009; 136: 486-95

- Drummond MF, Schulpher MJ, Torrance GW, et al. Methods for the Economic Evaluation of Health Care Programmes. 3rd ed. Oxford: Oxford University Press, 2005

- Kanwal F, Gralnek IM, Martin P, et al. Treatment alternatives for chronic hepatitis B virus infection: a cost-effectiveness analysis. Ann Intern Med 2005; 142: 821-31

- Buti M, Brosa M, Casado MA, et al. Modeling the cost-effectiveness of different oral antiviral therapies in patients with chronic hepatitis B. J Hepatol 2009; 51: 640-6

- Dakin H, Bentley A, Dusheiko G. Cost-Utility Analysis of Tenofovir Disoproxil Fumarate in the Treatment of Chronic Hepatitis B. Value Health 2010 Sep 3 [Epub ahead of print]

- Associazione Italiana di Economia Sanitaria (AIES). Proposta di linee guida per la valutazione economica degli interventi sanitari. PharmacoEconomics 2009; 11: 83-93

\section{Risposta dell'Autore}

Le critiche di Colombo e Viganò sono un contributo positivo che accettiamo nello spirito di far crescere la percezione che il decisore deve avere circa il significato da attribuire alle valutazioni farmacoeconomiche che vengono proposte nelle diverse pubblicazioni. Innanzitutto il nostro era un lavoro di revisione del valore clinico-terapeutico ed economico di entecavir alla luce dei dati disponibili a fine 2010-inizio 2011 e non aveva l'obiettivo di analizzare la costo-efficacia di tutte le alternative possibili nella terapia antivirale della HBV. In particolare, abbiamo voluto presentare i risultati della "Consensus Conference di Stresa 2007" che indicava entecavir e tenofovir come farmaci che hanno un profilo di efficacia tra loro equivalente e superiore a quella degli altri analoghi nucleosidici/nucleotidici, ma un profilo di tollerabilità sensibilmente differente a vantaggio di entecavir. Dopo la stesura del manoscritto sono stati pubblicati altri lavori che sostanzialmente hanno confermato il profilo di entecavir e l'equivalenza terapeutica con tenofovir. Il rischio di danno renale da tenofovir è stato riportato in casistiche limitate con risultati controversi.

Nella nostra rassegna abbiamo considerato essenzialmente il possibile impatto positivo che potrebbe indurre l'uso di entecavir sull'evoluzione clinica della epatite B e sui costi di gestione della malattia nella prospettiva del SSN. In questo contesto abbiamo citato un modello di simulazione markoviano, sviluppato e va- 
lidato da esperti internazionali della BMS e messo a nostra conoscenza ma non ancora pubblicato, del quale abbiamo riportato solo alcuni dettagli tecnici essenziali e solo alcuni risultati utili ai fini della valorizzazione dell'impatto economico indotto sul SSN dall'introduzione di entecavir. Questo modello non affrontava affatto il confronto tra entecavir e tenofovir, confronto che invece è stato oggetto principale di un'analisi costo-efficacia pubblicata da Colombo e coll. (2011), quando avevamo già licenziato le bozze del nostro lavoro.

Il modello markoviano della BMS che abbiamo utilizzato cercava di evidenziare come il costo di acquisto di entecavir potesse essere giustificato dal fatto che il valore dei benefici economici derivanti dal miglioramento della evoluzione dell'HBV era uguale o superiore al costo del trattamento. In quest'analisi non c'era confronto con tenofovir.

Nella parte finale della nostra rassegna, riconosciuto che il prezzo di acquisto di tenofovir è minore di quello di entecavir, abbiamo poi cercato di stimare se questa differenza potesse essere almeno in parte compensata dai minori costi delle reazioni avverse, senza alcuna pretesa di giungere a una conclusione definitiva, non avendo effettuato un'apposita valutazione di costo-efficacia. A tal proposito, l'analisi di Colombo e coll. conclude che tenofovir, nella terapia dell'HBV in Italia, è una strategia costo-efficace in confronto con tutti gli altri antivirali. Tuttavia, nei costi a carico del SSN italiano gli Autori non includono i costi delle reazioni avverse, ma si limitano a conteggiare il minimo costo del monitoraggio della funzione renale, previsto anche dalla scheda tecnica di tenofovir.

Siamo lieti che Colombo e Viganò concordino su una delle conclusioni fondamentali della nostra rassegna che recita «ad oggi, non è disponibile una valutazione precisa e robusta dell'impatto sul budget sanitario di entecavir vs. tenofovir, anche in virtù della mancanza di studi clinici controllati di confronto diretto tra $\mathrm{i}$ due farmaci», ma rigettiamo la critica che ci attribuisce un atteggiamento incongruente e partigiano a favore di entecavir.

Mario Eandi, Cattedra di Farmacologia Clinica, Facoltà di Medicina e Chirurgia, Università di Torino

\section{BIBLIOGRAFIA}

- Colombo GL, Gaeta GB, Viganò M, et al. A cost-effectiveness analysis of different therapies in patients with chronic hepatitis B in Italy. ClinicoEcon Outcomes Res 2011; 3: 37-46

- Gara N, Zhao X, Collins MT, et al. Renal tubular dysfunction during long-term adefovir or tenofovir therapy in chronic hepatitis B. Aliment Pharmacol Ther 2012; 35: 1317-25

- Gish RG, Clark MD, Kane SD, et al. Similar Risk of Renal Events Among Patients Treated With Tenofovir or Entecavir for Chronic Hepatitis B. Clin Gastroenterol Hepatol 2012; http://dx.doi.org/10.1016/j.cgh.2012.04.008

- Pol S, Lampertico P. First-line treatment of chronic hepatitis B with entecavir or tenofovir in "real-life" settings: from clinical trials to clinical practice. J Viral Hepatitis 2012; 19: 377-86

- Singal AK, Fontana RJ. Meta-analysis: oral anti-viral agents in adults with decompensated hepatitis B virus cirrhosis. Aliment Pharmacol Ther 2012; 35: 674-89 